\title{
Effect of one year treatment with inhaled fluticasone propionate or beclomethasone dipropionate on bone density and bone metabolism: a randomised parallel group study in adult asthmatic subjects
}

\author{
T C Medici, E Grebski, M Häcki, P Rüegsegger, C Maden, J Efthimiou, on behalf of a
} Swiss study group

\begin{abstract}
Background-There is some concern that prolonged treatment with high doses of inhaled corticosteroids may have a detrimental effect on bone mass. The aim of this one year study was to investigate the effects of low and high doses of fluticasone propionate (FP) $(400 \mu \mathrm{g} / \mathrm{day}$ and $750 \mu \mathrm{g} /$ day) and beclomethasone dipropionate (BDP) $(800 \mu \mathrm{g} / \mathrm{day}$ and $1500 \mu \mathrm{g} / \mathrm{day})$ on bone mass and metabolism.

Methods-This was a multicentre, double blind, parallel group study involving 69 mild to moderate asthmatic subjects who were randomised to treatment as follows: 22 to FP400, 21 to BDP800, 13 to FP750, and 13 to BDP1500. Their mean age was 39 years, $67 \%$ were men, and all the women were premenopausal.
\end{abstract}

Results-The results of peripheral quantitative computed tomographic (pQCT) measurements (primary variable) showed that, compared with baseline values, there was no loss of trabecular or integral (cortical and trabecular) bone in the distal radius or tibia in any of the patients over the 12 month study period. No consistent pattern emerged from the analysis of changes from baseline in markers of bone formation and resorption after six and 12 months of treatment.

Conclusion-The results of this study provide reassuring prospective one year data showing that inhaled corticosteroids, in the range of doses used, had no adverse effects on bone mass and metabolism in this group of asthmatic patients.

(Thorax 2000;55:375-382)

Keywords: fluticasone propionate; inhaled corticosteroids; bone mineral density; bone metabolism; asthma

Wellcome R\&D,

Stockley Park, UK

C Maden

J Efthimiou

Correspondence to: Professor T C Medici

Received 11 January 1999 Returned to author Returned to a
8 April 1999

Revised manuscript received

20 December 1999

Accepted for publication

20 January 2000

Prolonged treatment with oral corticosteroids is associated with a reduction in bone density, osteoporosis, and a risk of fractures. ${ }^{1}$ Inhaled corticosteroids have provided a major breakthrough in the treatment of asthma, producing improvements in symptoms and lung function without the risk of serious side effects commonly associated with oral corticosteroids. There is some concern, however, that pro- longed treatment with high doses of inhaled corticosteroids may have a detrimental effect on bone mass. ${ }^{2}$

Several studies have reported changes in bone mineral density (BMD) in asthmatic patients using high dose inhaled corticosteroids. $^{3-10}$ However, most of these studies have been uncontrolled and cross sectional in design. In many cases, too, the results have been confounded by previous or concomitant use of oral steroids or by other factors known to influence bone density such as the menopause. Changes in biochemical markers of bone metabolism have also been reported in normal subjects receiving inhaled corticosteroids over short periods. ${ }^{11-14}$ However, the results from clinical studies are less convincing. Whereas a few studies have found significant effects on bone metabolism, ${ }^{15} 16$ substantially more, including long term studies, have reported no or only a small effect. ${ }^{17}{ }^{18}$ Clearly, further long term research is needed to investigate the effects of inhaled corticosteroids on both bone density and bone metabolism in asthmatic patients.

Fluticasone propionate (FP) is a recently available inhaled corticosteroid ${ }^{19}$ with negligible oral bioavailability ${ }^{20}$ and therefore a reduced potential for systemic effects. A number of clinical studies have shown that FP has an improved therapeutic ratio when compared with other inhaled corticosteroids, ${ }^{21}$ and at half the microgram dose is at least as effective as beclomethasone dipropionate (BDP) and budesonide. $^{22-27}$

The aim of this study was to investigate prospectively and compare the effects of treatment with low and high doses of inhaled FP and BDP over a one year period on BMD and biochemical markers of bone metabolism in adult patients with moderately severe asthma.

\section{Methods}

SUBJECTS
Patients with mild to moderate asthma were recruited from the outpatient departments of seven centres in Switzerland. The age limit was 20-55 years for men and 20-45 years for women (premenopausal). For the six months preceding the start of the study patients had been receiving regular treatment with inhaled corticosteroids in doses ranging from 400 to $1600 \mu \mathrm{g} /$ day. 
Patients were excluded from the study for the following reasons: a change in regular asthma medication (other than inhaled corticosteroids), treatment with antibiotics for infections of the upper or lower respiratory tract, admission to hospital during the previous four weeks; treatment with systemic corticosteroids during the previous eight weeks; more than three short courses of oral steroids or depot corticosteroids in the previous 12 months; excessively overweight or underweight; immobilisation; fractures occurring within the six months preceding the start of the study; disorders of bone metabolism such as osteoporosis or Paget's disease; pregnancy, lactation, inadequate contraceptive precautions, amenorrhoea or a history of irregular menstrual cycles during the 12 months preceding the start of the study; treatment with any medication likely to influence bone metabolism.

The study was approved by the appropriate local ethics committee and all subjects provided written informed consent. The study was performed in accordance with the principles of the Declaration of Helsinki and with Good Clinical Practice.

\section{STUDY DESIGN}

This was a multicentre, double blind, randomised, parallel group study of 12 months duration. After an initial screening visit patients entered a four week run in period during which their regular inhaled corticosteroid therapy was standardised to either BDP $800 \mu \mathrm{g} /$ day or $1500 \mu \mathrm{g} / \mathrm{day}$, depending on the dose of their inhaled corticosteroid prior to entry and at the discretion of the investigator. Salbutamol metered dose inhaler was used as required to relieve symptoms and most patients also used long acting $\beta_{2}$ agonists.

After successful completion of this period, and depending upon whether they were in the low (BDP800) or high (BDP1500) dose run in group, patients were randomly assigned to treatment with either fluticasone propionate (FP) $400 \mu \mathrm{g} /$ day $(200 \mu \mathrm{g}$ twice daily) or BDP $800 \mu \mathrm{g} /$ day $(400 \mu \mathrm{g}$ twice daily), or FP $750 \mu \mathrm{g} /$ day $(375 \mu \mathrm{g}$ twice a day) or BDP $1500 \mu \mathrm{g} /$ day

Table 1 Baseline characteristics

\begin{tabular}{|c|c|c|c|c|}
\hline & $\begin{array}{l}F P 400 \\
(n=22)\end{array}$ & $\begin{array}{l}B D P 800 \\
(n=21)\end{array}$ & $\begin{array}{l}F P 750 \\
(n=13)\end{array}$ & $\begin{array}{l}B D P 1500 \\
(n=13)\end{array}$ \\
\hline Men & $17(77 \%)$ & $13(62 \%)$ & $9(69 \%)$ & $7(54 \%)$ \\
\hline Women & $5(23 \%)$ & $8(38 \%)$ & $4(31 \%)$ & $6(46 \%)$ \\
\hline Age $(\text { years })^{\star}$ & $39(8)$ & $38(8)$ & $38(10)$ & $40(10)$ \\
\hline Caucasian & $21(95 \%)$ & $21(100 \%)$ & $12(92 \%)$ & $12(92 \%)$ \\
\hline Height $(\mathrm{cm})^{\star}$ & $174(6)$ & $171(10)$ & $172(8)$ & $170(8)$ \\
\hline Weight $(\mathrm{kg})^{\star}$ & $75(10)$ & $70(14)$ & $74(13)$ & $64(12)$ \\
\hline \multicolumn{5}{|l|}{ Baseline } \\
\hline \multicolumn{4}{|l|}{ Baseline } & $78.2(14.8)$ \\
\hline$\%$ Predicted clinic $\mathrm{PEF}^{\star}$ & $88.7(15.1)$ & $97.8(17.2)$ & $78.4(30.2)$ & $89.6(27.1)$ \\
\hline \multicolumn{5}{|l|}{ Duration of asthma (n) } \\
\hline$<12$ years & 2 & 0 & 0 & 0 \\
\hline$\geqslant 12$ years & 20 & 21 & 13 & 13 \\
\hline \multicolumn{5}{|l|}{ History of smoking, $\mathrm{n}(\%)$} \\
\hline Never & $11(50 \%)$ & $13(62 \%)$ & $6(46 \%)$ & $6(46 \%)$ \\
\hline Ex-smoker & $8(36 \%)$ & $7(38 \%)$ & $4(31 \%)$ & $4(31 \%)$ \\
\hline Current & $3(14 \%)$ & $1(5 \%)$ & $3(23 \%)$ & $3(23 \%)$ \\
\hline
\end{tabular}

FP400 = fluticasone propionate $400 \mu \mathrm{g} /$ day $(200 \mu \mathrm{g}$ bd); FP750 = fluticasone propionate $750 \mu \mathrm{g} /$ day $(375 \mu \mathrm{g} \mathrm{bd}) ;$ BDP800 = beclomethasone dipropionate $800 \mu \mathrm{g} /$ day $(400 \mu \mathrm{g} \mathrm{bd}) ; \mathrm{BDP} 1500=$ beclomethasone dipropionate $1500 \mu \mathrm{g} /$ day $(750 \mu \mathrm{g} \mathrm{bd}) ; \mathrm{FEV}_{1}=$ forced expiratory volume in one second; PEF = peak expiratory flow.

$\star$ Values are mean $(\mathrm{SD})$.
(750 $\mu \mathrm{g}$ twice a day). Study treatment was administered using a metered dose inhaler (MDI) and a large volume spacer device (Volumatic $\left.^{\mathrm{TM}}\right)$. All other asthma medication remained unchanged.

Clinic visits were held at the beginning and end of the run in period and every two months thereafter throughout the 12 month treatment period. Patients attended an additional follow up visit two weeks after completing the study.

\section{PROCEDURE}

At the initial screening visit patients underwent a full physical examination and provided details of their medical history. The physical examination was repeated at the final 12 month visit.

Samples of venous blood and urine were collected at the start (0), mid point (6 months), and end (12 months) of treatment. Blood samples were taken from fasted patients between 08.00 and 10.00 hours. Urine samples were taken from 24 hour collections started at 08.00 hours on the day before the clinic visit and preserved in hydrochloric acid. Blood and urine samples taken at the start and end of treatment were analysed for routine haematological and biochemical parameters. Serum samples taken at 0,6 , and 12 months were analysed for cortisol and markers of bone metabolism including osteocalcin, procollagen type I carboxy terminal propeptide (PICP), alkaline phosphatase (bone specific), creatinine, calcium, and carboxy terminal cross linked telopeptide of type I collagen (ICTP). Urine samples taken at 0,6 , and 12 months were analysed for calcium, phosphate, creatinine, hydroxyproline, and deoxypyridinoline. All samples were analysed centrally by the Department of Clinical Chemistry, University Hospital, Zurich.

The cortisol samples were analysed using the Immulite TM (DPC) chemoluminescence immunoassay. Osteocalcin, PICP, and ICTP were analysed using radioimmunoassay and hydroxyproline was analysed by high performance liquid chromatography (HPLC). Deoxypyridinoline was analysed by enzyme immunoassay.

BMD was measured at 0,6 , and 12 months using peripheral quantitative computed tomography (pQCT) and dual energy $x$ ray absorptiometry (DXA). pQCT (Densiscan-1000, Scanco Medical, Switzerland) was used to measure the BMD $\left(\mathrm{mg} / \mathrm{cm}^{3}\right)$ of the radius and tibia, evaluating trabecular, total (integral), and compact bone. For trabecular bone the results were expressed as hydroxyapatite densities $\left(\mathrm{HA} / \mathrm{cm}^{3}\right)$. To obtain these, the European forearm phantom ${ }^{28}$ (containing standardised amounts of 50,100 , and $200 \mathrm{mg} / \mathrm{cm}^{3}$ hydroxyapatite) was regularly measured during the study and the bone densities converted to hydroxyapatite densities. Precision of the pQCT system is $0.3 \%$ relative to normal bone densities in the case of trabecular bone and 1\% in the case of compact bone. DXA (Lunar DPX-L scanner, Lunar Corporation, Madison, Wisconsin, USA) was used to measure BMD $\left(\mathrm{mg} / \mathrm{cm}^{2}\right)$ of the lumbar spine in an anteriorposterior direction, evaluating a mixture of cortical and trabecular bone. To obtain 
Table 2 Radial and tibial distal bone mineral density (BMD) measurements

\begin{tabular}{|c|c|c|c|c|c|c|}
\hline & $\begin{array}{l}F P 400 \\
m g H A / \mathrm{cm}^{3} \\
(n=22)\end{array}$ & $\begin{array}{l}\text { BDP800 } \\
\mathrm{mgHA} / \mathrm{cm}^{3} \\
(n=21)\end{array}$ & $\begin{array}{l}p \text { value } \\
F P-B D P^{*}\end{array}$ & $\begin{array}{l}F P 750 \\
m g H A / \mathrm{cm}^{3} \\
(n=13)\end{array}$ & $\begin{array}{l}\mathrm{BDP} 1500 \\
\mathrm{mgHA} / \mathrm{cm}^{3} \\
(n=13)\end{array}$ & $\begin{array}{l}p \text { value } \\
F P-B D P^{*}\end{array}$ \\
\hline \multicolumn{7}{|l|}{ Radius } \\
\hline \multicolumn{7}{|l|}{ Trabecular BMD } \\
\hline Baseline median & 131 & 156 & & 142 & 109 & \\
\hline Interquartile range & $117-174$ & $132-185$ & & $118-162$ & $85-131$ & \\
\hline \multicolumn{7}{|l|}{$6 \mathrm{~m}$} \\
\hline Median change & 0.5 & 1.0 & NS & 1.0 & 0.0 & NS \\
\hline Quartile change & $-1.0,2.0$ & $-0.5,1.0$ & & $0.0,1.0$ & $-1.0,1.00$ & \\
\hline \multicolumn{7}{|l|}{$12 \mathrm{~m}$} \\
\hline Median change & 1.0 & 1.0 & NS & 0.0 & 0.0 & NS \\
\hline Quartile change & $-1.0,2.0$ & $-2.5,-1.5$ & & $-1.0,1.0$ & $-1.5,1.0$ & \\
\hline \multicolumn{7}{|l|}{ Integral BMD } \\
\hline Baseline median & 328 & 365 & & 327 & 349 & \\
\hline Interquartile range & $285-385$ & $344-399$ & & $310-339$ & $288-364$ & \\
\hline \multicolumn{7}{|l|}{$6 \mathrm{~m}$} \\
\hline Median change & 0.0 & 1.0 & NS & 0.0 & & NS \\
\hline Quartile change & $-1.0,3.0$ & $-2.0,1.5$ & & $-1.5,1.5$ & $-1.0,1.0$ & \\
\hline \multicolumn{7}{|l|}{$12 \mathrm{~m}$} \\
\hline Median change & 1.0 & -0.5 & NS & 0.0 & 1.0 & NS \\
\hline Quartile change & $0.0,2.0$ & $-2.0,3.0$ & & $-2.0,1.5$ & $-1.0,2.0$ & \\
\hline \multicolumn{7}{|l|}{ Compact BMD } \\
\hline Baseline median & 767 & 817 & & 765 & 802 & \\
\hline Interquartile range & $719-853$ & $774-866$ & & $730-795$ & $770-834$ & \\
\hline \multicolumn{7}{|l|}{$6 \mathrm{~m}$} \\
\hline Median change & -0.5 & 0.0 & NS & -1.5 & -1.0 & NS \\
\hline Quartile change & $-4.0,5.0$ & $-2.0,3.0$ & & $-4.0,3.0$ & $-3.0,0.0$ & \\
\hline \multicolumn{7}{|l|}{$12 \mathrm{~m}$} \\
\hline Median change & 0.0 & 1.5 & NS & -1.5 & & 0.048 \\
\hline Quartile change & $-3.0,4.0$ & $-2.0,4.0$ & & $-3.5,0.0$ & $-0.5,3.0$ & \\
\hline \multicolumn{7}{|l|}{ Tibia } \\
\hline \multicolumn{7}{|l|}{ Trabecular BMD } \\
\hline Baseline median & 141 & 129 & & 140 & 102 & \\
\hline Interquartile range & $104-174$ & $103-168$ & & $125-173$ & $88-117$ & \\
\hline \multicolumn{7}{|l|}{$6 \mathrm{~m}$} \\
\hline Median change & 0.5 & -0.5 & NS & 0.0 & & NS \\
\hline Quartile change & $-1.0,2.0$ & $-2.0,0.0$ & & $-0.5,1.0$ & $0.0,0.0$ & \\
\hline \multicolumn{7}{|l|}{$12 \mathrm{~m}$} \\
\hline Median change & 0.0 & 0.0 & NS & 0.0 & 0.0 & NS \\
\hline Quartile change & $-1.0,1.0$ & $-2.0,1.0$ & & $-0.5,1.5$ & $-0.5,1.0$ & \\
\hline \multicolumn{7}{|l|}{ Integral BMD } \\
\hline Baseline median & 312 & 300 & & 316 & 281 & \\
\hline Interquartile range & $281-349$ & $274-354$ & & $290-352$ & $244-304$ & \\
\hline \multicolumn{7}{|l|}{$6 \mathrm{~m}$} \\
\hline Median change & 0.0 & -0.5 & NS & -0.5 & 0.0 & NS \\
\hline Quartile change & $-1.0,1.0$ & $-1.5,1.0$ & & $-1.5,0.5$ & $-1.0,1.0$ & \\
\hline \multicolumn{7}{|l|}{$12 \mathrm{~m}$} \\
\hline Median change & 0.0 & 0.0 & NS & 0.0 & & NS \\
\hline Quartile change & $-1.0,2.0$ & $-1.0,2.5$ & & $-1.5,1.0$ & $-2.0,1.5$ & \\
\hline Compact BMD & & & & & & \\
\hline Baseline median & 579 & 601 & & 565 & 571 & \\
\hline Interquartile range & $544-662$ & $545-675$ & & $530-626$ & $500-610$ & \\
\hline $6 \mathrm{~m}$ & & & & & & \\
\hline Median change & 0.5 & -0.5 & NS & -1.0 & 0.0 & NS \\
\hline Quartile change & $-1.0,4.0$ & $-4.0,2.0$ & & $-3.0,1.5$ & $-3.0,1.0$ & \\
\hline $12 \mathrm{~m}$ & & & & & & \\
\hline Median change & 1.0 & -0.5 & NS & -1.5 & & NS \\
\hline Quartile change & $-2.0,-4.0$ & $-2.0,4.0$ & & $-3.5,1.0$ & $-4.0,2.0$ & \\
\hline
\end{tabular}

FP400 = fluticasone propionate $400 \mu \mathrm{g} / \mathrm{day}(200 \mu \mathrm{g}$ bd); FP750 = fluticasone propionate $750 \mu \mathrm{g} / \mathrm{day}(375 \mu \mathrm{g}$ bd); BDP800 = beclomethasone dipropionate $800 \mu \mathrm{g} / \mathrm{day}(400 \mu \mathrm{g} \mathrm{bd}) ; \mathrm{BDP} 1500=$ beclomethasone dipropionate $1500 \mu \mathrm{g} / \mathrm{day}(750 \mu \mathrm{g} \mathrm{bd}) ; \mathrm{m}=$ months.

${ }^{\star} \mathrm{p}$ value $\mathrm{FP}-\mathrm{BDP}=$ significance derived from Wilcoxon rank sum test (difference between treatments in change from baseline).

standardised results the European spine phantom ${ }^{29}$ (containing standardised amounts of 50,100 , and $200 \mathrm{mg} / \mathrm{cm}^{3}$ hydroxyapatite) was continuously measured during the study. Precision of the DXA system was $1 \%$ relative to normal BMD values.

All the measurements were performed at the Institute of Biomedical Engineering, University of Zurich. All scans were performed under 'blinded' conditions. In patients prescribed a course of oral steroids the bone scan was performed four weeks after the course was completed.

Subjects were issued with a mini Wright peak flow meter and shown how to complete daily record cards of morning and evening peak expiratory flow (PEF). These diary cards were completed before taking study medication, every day during the last two weeks of the run in period and thereafter during the two weeks preceding each clinic visit. Lung function (forced expiratory volume in one second, $\mathrm{FEV}_{1}$ ) was measured at each clinic visit and patients were asked not to use any salbutamol for at least four hours beforehand. Where possible, $\mathrm{FEV}_{1}$ assessments were made at the same time of day, preferably in the morning, with the highest of three values being recorded.

Adverse events and any changes in concurrent medication were recorded at every clinic visit. An oropharyngeal assessment to test for the presence of oral candidiasis was performed at all clinic visits during treatment.

ANALYSIS OF DATA

Taking the standard deviation of 1.55 for percentage change in trabecular BMD obtained in a previous pQCT study, ${ }^{30} 92$ 
Table 3 Radial and tibial distal bone mineral density (BMD) measurements expressed as mean (SD) $Z$ scores

\begin{tabular}{|c|c|c|c|c|}
\hline & $\begin{array}{l}F P 400 \\
(n=22)\end{array}$ & $\begin{array}{l}\text { BDP800 } \\
(n=21)\end{array}$ & $\begin{array}{l}F P 750 \\
(n=13)\end{array}$ & $\begin{array}{l}B D P 1500 \\
(n=13)\end{array}$ \\
\hline \multicolumn{5}{|c|}{ Radius } \\
\hline \multicolumn{5}{|c|}{ Trabecular BMD } \\
\hline Baseline & $-0.145(1.450)$ & $0.152(0.825)$ & $-0.230(1.106)$ & $-0.517(1.320)$ \\
\hline $12 \mathrm{~m}$ & $-0.078(1.472)$ & $0.097(0.852)$ & $-0.216(1.125)$ & $-0.432(1.337)$ \\
\hline \multicolumn{5}{|c|}{ Integral BMD } \\
\hline Baseline & $0.018(1.349)$ & $0.558(1.023)$ & $-0.094(1.007)$ & $0.013(1.207)$ \\
\hline $12 \mathrm{~m}$ & $0.042(1.395)$ & $0.583(1.076)$ & $-0.063(1.025)$ & $0.093(1.235)$ \\
\hline \multicolumn{5}{|c|}{ Compact BMD } \\
\hline Baseline & $0.174(1.441)$ & $0.603(1.194)$ & $-0.170(1.217)$ & $0.121(1.187)$ \\
\hline $12 \mathrm{~m}$ & $0.037(1.358)$ & $0.652(1.217)$ & $-0.234(1.233)$ & $0.200(1.233)$ \\
\hline \multicolumn{5}{|c|}{ Tibia } \\
\hline \multicolumn{5}{|c|}{ Trabecular BMD } \\
\hline Baseline & $-0.251(1.066)$ & $-0.350(0.906)$ & $-0.115(1.079)$ & $-0.627(1.081)$ \\
\hline $12 \mathrm{~m}$ & $-0.184(1.036)$ & $-0.437(0.874)$ & $0.034(0.977)$ & $-0.526(1.049)$ \\
\hline \multicolumn{5}{|c|}{ Integral BMD } \\
\hline Baseline & $-0.086(1.047)$ & $-0.249(0.824)$ & $-0.046(0.724)$ & $-0.555(1.188)$ \\
\hline $12 \mathrm{~m}$ & $-0.039(1.057)$ & $-0.266(0.844)$ & $0.024(0.688)$ & $-0.469(1.198)$ \\
\hline \multicolumn{5}{|c|}{ Compact BMD } \\
\hline Baseline & $-0.138(1.291)$ & $0.131(0.912)$ & $-0.320(1.090)$ & $-0.503(1.001)$ \\
\hline $12 \mathrm{~m}$ & $-0.124(1.337)$ & $0.125(0.952)$ & $-0.243(1.082)$ & $-0.441(1.013)$ \\
\hline
\end{tabular}

FP400 = fluticasone propionate $400 \mu \mathrm{g} /$ day $(200 \mu \mathrm{g}$ bd $) ; \mathrm{FP} 750=$ fluticasone propionate $750 \mu \mathrm{g}$ day $(375 \mu \mathrm{g} \mathrm{bd}) ; \mathrm{BDP} 800=$ beclomethasone dipropionate $800 \mu \mathrm{g} /$ day $(400 \mu \mathrm{g} \mathrm{bd}) ; \mathrm{BDP} 1500=$ beclomethasone dipropionate $1500 \mu \mathrm{g} / \mathrm{day}(750 \mu \mathrm{g}$ bd).

evaluable subjects (23 per treatment group) were required to ensure a power of $80 \%$ to detect a $1.3 \%$ difference between treatments in change from baseline.

Analysis was performed on the intent-totreat population. The primary safety parameter was BMD of the distal radius measured using pQCT.

Differences between treatments in changes from baseline in BMD of trabecular, total, and compact bone as measured by pQCT and $\mathrm{BMD}$ of lumbar vertebrae as measured by DXA were analysed using the Wilcoxon rank sum test. Similar methods of analysis were applied to bone markers. All statistical tests performed were two sided with $\mathrm{p}$ values of 0.05 considered significant.

$\mathrm{Z}$ scores, representing the number of standard deviations above or below the statistical mean of a reference population (Rüegsegger, data on file) taking into account age and sex, were calculated and summarised for BMD of trabecular, total, and compact bone of the radius and tibia.

No formal analysis was applied to serum cortisol, daily diary card data (PEF, symptoms scores or use of additional bronchodilator), or clinic lung function data.

\section{Results}

SUBJECT CHARACTERISTICS

Of the 69 subjects randomised to treatment, 43 were assigned to the low dose comparison group (22 to FP400, 21 to BDP800) and 26 to the high dose comparison group (13 to FP750, 13 BDP1500). Demographic and baseline characteristics were well matched in patients in both treatment comparisons (table 1). The age range was $22-55$ years with a mean age of 39 years and a preponderance of men $(67 \%)$. All the women were premenopausal. No patient had a family history of fracture or vertebral crush or wedge fractures. One patient had suffered a wrist fracture and seven patients reported other fractures, none of them of the femoral neck. Patients in the low dose comparison had used on average $745 \mu \mathrm{g} /$ day inhaled corticosteroid during the previous 12 months and patients in the high dose group had taken approximately $1260 \mathrm{mg}$ /day.

Four subjects were withdrawn from the study, one from each of the four treatment groups. Reasons for withdrawal were as follows: adverse event and non-compliance (BDP $1500 \mu \mathrm{g} /$ day and BDP $800 \mu \mathrm{g} /$ day, respectively); no reason specified (FP $400 \mu \mathrm{g} /$ day and FP $750 \mu \mathrm{g} /$ day).

Four patients required a short course of oral corticosteroid during the study for the treatment of an asthma exacerbation (one taking BDP $800 \mu \mathrm{g} /$ day, two taking FP $750 \mu \mathrm{g} /$ day, and one taking BDP $1500 \mu \mathrm{g} /$ day).

\section{BONE MINERAL DENSITY}

There was no significant difference in change from baseline in BMD of the distal radius measured using $\mathrm{pQCT}$ for either of the two treatment comparisons at six or 12 months. Overall, the results of pQCT measurement showed that, compared with baseline values, there was no loss of trabecular or integral bone in the radius or tibia in any of the patients over the 12 month study period (table 2). While some negative changes were recorded in the median bone density of compact bone of the radius (patients taking $\mathrm{FP} 750 \mu \mathrm{g} /$ day) and tibia (patients taking BDP $800 \mu \mathrm{g} /$ day and FP $750 \mu \mathrm{g} /$ day), none of these changes exceeded $-2 \%$ which suggests that the results were not clinically significant.

From the non-parametric analyses, the only result of borderline significance was derived from the high dose comparison of compact bone density of the radius at 12 months $(\mathrm{p}=$ 0.048 ) in patients taking FP $750 \mu \mathrm{g} /$ day and BDP $1500 \mu \mathrm{g} /$ day (table 2). While the decrease in bone density was greater in patients taking FP $750 \mu \mathrm{g} /$ day, negative changes in bone density were recorded in just three of the 12

Table 4 Bone mineral density $\left(\mathrm{g} / \mathrm{cm}^{2}\right)$ of lumbar spine

\begin{tabular}{|c|c|c|c|c|c|c|}
\hline & $\begin{array}{l}F P 400 \\
(n=22)\end{array}$ & $\begin{array}{l}B D P 800 \\
(n=21)\end{array}$ & $\begin{array}{l}p \text { value } \\
F P-B D P^{*}\end{array}$ & $\begin{array}{l}F P 750 \\
(n=13)\end{array}$ & $\begin{array}{l}B D P 1500 \\
(n=13)\end{array}$ & $\begin{array}{l}p \text { value } \\
F P-B D P^{*}\end{array}$ \\
\hline Baseline median & 1.26 & 1.14 & & 1.25 & 1.14 & \\
\hline Interquartile range & $1.05-1.33$ & $1.08-1.19$ & & $1.09-1.35$ & $1.05-1.19$ & \\
\hline \multicolumn{7}{|l|}{6 months } \\
\hline Median change & 0.005 & 0.005 & NS & -0.004 & 0.0 & NS \\
\hline Quartile change & $-0.004,0.022$ & $-0.013,0.020$ & & $-0.031,0.019$ & $-0.011,0.010$ & \\
\hline \multicolumn{7}{|l|}{12 months } \\
\hline Median change & 0.020 & -0.003 & 0.02 & 0.005 & -0.005 & NS \\
\hline Quartile change & $-0.005,0.033$ & $-0.016,0.009$ & & $-0.023,0.019$ & $-0.018,0.021$ & \\
\hline
\end{tabular}

FP400 = fluticasone propionate $400 \mu \mathrm{g} /$ day $(200 \mu \mathrm{g} \mathrm{bd}) ; \mathrm{FP} 750=$ fluticasone propionate $750 \mu \mathrm{g} / \mathrm{day}(375 \mu \mathrm{g}$ bd $) ; \mathrm{BDP} 800=$ beclomethasone dipropionate $800 \mu \mathrm{g} /$ day $(400 \mu \mathrm{g} \mathrm{bd}) ; \mathrm{BDP} 1500=$ beclomethasone dipropionate $1500 \mu \mathrm{g} / \mathrm{day}(750 \mu \mathrm{g} \mathrm{bd})$. ${ }^{\star} \mathrm{p}$ value $\mathrm{FP}-\mathrm{BDP}=$ significance derived from Wilcoxon rank sum test (difference between treatments in change from baseline). 
Table 5 Biochemical markers of bone formation and resorption

\begin{tabular}{|c|c|c|c|c|c|c|c|}
\hline & $\begin{array}{l}\text { Reference } \\
\text { range }\end{array}$ & $\begin{array}{l}F P 400 \\
(n=22)\end{array}$ & $\begin{array}{l}\text { BDP800 } \\
(n=20)\end{array}$ & $\begin{array}{l}p \text { value } \\
F P-B D P^{\star}\end{array}$ & $\begin{array}{l}F P 750 \\
(n=13)\end{array}$ & $\begin{array}{l}B D P 1500 \\
(n=13)\end{array}$ & $\begin{array}{l}p \text { value } \\
F P-B D P^{\star}\end{array}$ \\
\hline \multicolumn{8}{|l|}{ Bone formation: } \\
\hline Osteocalcin $(\mu \mathrm{g} / \mathrm{l})$ & $2.4-11.7$ & 5.3 & 5.5 & & 4.7 & 5.6 & \\
\hline Interquartile range & & $4.2-5.8$ & $4.8-6.2$ & & $4.0-5.4$ & $3.3-7.2$ & \\
\hline \multicolumn{8}{|l|}{6 months } \\
\hline Median change & & -0.30 & 0.30 & NS & 0.95 & 0.30 & NS \\
\hline Quartile change & & $-1.40,1.60$ & $-1.00,2.20$ & & $-0.55,1.75$ & $-1.40,1.10$ & \\
\hline \multicolumn{8}{|l|}{12 months } \\
\hline Median change & & 0.10 & -1.05 & 0.047 & -0.45 & -0.60 & NS \\
\hline Quartile change & & $-1.40,0.90$ & $-1.80,-0.20$ & & $-0.80,0.80$ & $-1.85,0.17$ & \\
\hline $\operatorname{PICP}(\mu \mathrm{g} / \mathrm{l})$ & $40-200$ & 117 & 134 & & 112 & 136 & \\
\hline Interquartile range & & $92-131.0$ & $102-170$ & & $92-131$ & $108-161$ & \\
\hline \multicolumn{8}{|l|}{6 months } \\
\hline Median change & & -5.7 & -8.5 & NS & 6.0 & -9.0 & NS \\
\hline Quartile change & & $-25.0,12.3$ & $-35.0,7.0$ & & $2.0,34.0$ & $-18.0,8.0$ & \\
\hline 12 months & & & & & & & \\
\hline Median change & & 5.5 & -4.0 & NS & 7.5 & 1.5 & NS \\
\hline Quartile change & & $-11.6,15.5$ & $-29.0,8.0$ & & $-17.0,21.0$ & $-37.0,9.5$ & \\
\hline Serum alkaline phosphatase $(\mu / 1)$ & $<65$ & 20 & 22 & & 20 & 19 & \\
\hline Interquartile range & & $14-28$ & $19-28$ & & $14-25$ & $16-25$ & \\
\hline 6 months & & & & & & & \\
\hline Median change & & 0.0 & -1.5 & NS & -4.0 & 3.5 & NS \\
\hline Quartile change & & $-3.0,4.0$ & $-5.0,2.0$ & & $-9.0,5.0$ & $-4.0,8.5$ & \\
\hline 12 months & & & & & & & \\
\hline Median change & & 4.0 & 3.5 & NS & 3.0 & -1.0 & NS \\
\hline Quartile change & & $0.0,6.0$ & $-1.5,9.0$ & & $-1.0,5.0$ & $-3.5,6.5$ & \\
\hline Serum creatinine $(\mu \mathrm{mol} / \mathrm{l})$ & M70-105 & 94 & 94 & & 96 & 92 & \\
\hline Interquartile range & F60-90 & $83-102$ & $83-100$ & & $89-102$ & $82-96$ & \\
\hline 6 months & & & & & & & \\
\hline Median change & & 0.0 & 0.0 & NS & -0.5 & -2.0 & NS \\
\hline Quartile change & & $-4.0,6.0$ & $-5.5,3.5$ & & $-3.0,4.0$ & $-7.0,0.0$ & \\
\hline 12 months & & & & & & & \\
\hline Median change & & -1.0 & 0.0 & NS & -3.0 & -2.0 & NS \\
\hline Quartile change & & $-6.0,3.0$ & $-4.0,5.0$ & & $-6.0,0.0$ & $-7.0,0.5$ & \\
\hline Serum calcium $(\mathrm{mmol} / \mathrm{l})$ & $2.1-2.6$ & 2.33 & 2.30 & & 2.33 & 2.31 & \\
\hline Interquartile range & & $2.28-2.38$ & $2.28-2.33$ & & $2.27-2.38$ & $2.29-2.35$ & \\
\hline 6 months & & & & & & & \\
\hline Median change & & -0.005 & -0.015 & NS & 0.020 & 0.020 & NS \\
\hline Quartile change & & $-0.050,0.080$ & $-0.060,0.040$ & & $-0.040,0.055$ & $-0.030,0.040$ & \\
\hline 12 months & & & & & & & \\
\hline Median change & & -0.010 & 0.000 & NS & -0.005 & 0.060 & NS \\
\hline Quartile change & & $-0.050,0.070$ & $-0.040,0.030$ & & $-0.060,0.060$ & $-0.005,0.085$ & \\
\hline Bone resorption: & & & & & & & \\
\hline ICTP $(\mu \mathrm{g} / 1)$ & $1.8-5.0$ & 2.5 & 3.0 & & 2.5 & 2.5 & \\
\hline Interquartile range & & $1.9-2.8$ & $2.4-3.4$ & & $2.4-3.4$ & $2.3-2.9$ & \\
\hline 6 months & & & & & & & \\
\hline Median change & & -0.05 & -0.40 & NS & 0.30 & -0.30 & 0.031 \\
\hline Quartile change & & $-0.50,0.40$ & $-0.8,0.05$ & & $0.00,0.50$ & $-0.60,0.10$ & \\
\hline 12 months & & & & & & & \\
\hline Median change & & -0.30 & -0.20 & NS & -0.30 & -0.50 & NS \\
\hline Quartile change & & $-0.70,0.20$ & $-0.90,0.10$ & & $-0.75,0.4$ & $-0.70,-0.05$ & \\
\hline Hydroxyproline $(\mu \mathrm{mol} / \mathrm{l})$ & $100-400$ & 143 & 137 & & 184 & 116 & \\
\hline Interquartile range & & $116-228$ & $108-198$ & & $128-212$ & $96-166$ & \\
\hline 6 months & & & & & & & \\
\hline Median change & & 16 & 6 & NS & 27 & -21 & NS \\
\hline Quartile change & & $-48,62$ & $-35,41$ & & $-70,69$ & $-48,2$ & \\
\hline 12 months & & & & & & & \\
\hline Median change & & 45 & 22 & NS & 31 & -8 & NS \\
\hline Quartile change & & $-26,120$ & $-41,69$ & & $-74,51$ & $-63,30$ & \\
\hline Urine calcium $(\mathrm{mmol} / \mathrm{l})$ & $1.0-7.0$ & 3.92 & 3.39 & & 3.13 & 2.10 & \\
\hline Interquartile range & & $2.58,4.71$ & $2.12,5.98$ & & $1.79,4.34$ & $1.22,2.17$ & \\
\hline 6 months & & & & & & & \\
\hline Median change & & -0.27 & 0.18 & NS & 0.22 & 0.23 & NS \\
\hline Quartile change & & $-0.77,1.10$ & $-1.60,1.00$ & & $-0.88,0.60$ & $-0.15,0.84$ & \\
\hline 12 months & & & & & & & \\
\hline Median change & & -0.07 & 0.04 & NS & -0.05 & 0.26 & NS \\
\hline Quartile change & & $-1.71,1.60$ & $-2.15,1.73$ & & $-1.78,0.93$ & $-0.22,0.90$ & \\
\hline Urine phosphate $(\mathrm{mmol} / \mathrm{l})$ & $15-45$ & 24.5 & 27.8 & & 23.9 & 12.0 & \\
\hline Interquartile range & & $16.8-31.5$ & $15.4-35.4$ & & $15.9-29.9$ & $9.4-15.0$ & \\
\hline 6 months & & & & & & & \\
\hline Median change & & -0.3 & -2.2 & NS & 2.8 & -2.2 & NS \\
\hline Quartile change & & $-4.2,3.8$ & $-12.2,5.0$ & & $-9.6,13.6$ & $-4.2,0.2$ & \\
\hline 12 months & & & & & & & \\
\hline Median change & & -1.6 & -4.2 & NS & 2.0 & -1.7 & NS \\
\hline Quartile change & & $-6.6,6.0$ & $-15.8,3.4$ & & $-16.0,5.8$ & $-6.2,2.6$ & \\
\hline Deoxypyridinoline:creatinine ratio & $2.5-9.5$ & 3.7 & 3.9 & & 4.4 & 3.9 & \\
\hline Interquartile range & & $3.4-4.4$ & $3.6-5.4$ & & $3.4-4.8$ & $3.5-5.7$ & \\
\hline 6 months & & & & & & & \\
\hline Median change & & 0.05 & 0.10 & NS & 0.80 & 0.00 & NS \\
\hline Quartile change & & $-0.80,0.80$ & $-1.30,0.35$ & & $-0.90,1.55$ & $-0.80,0.80$ & \\
\hline 12 months & & & & & & & \\
\hline Median change & & -0.10 & -0.65 & NS & 0.00 & 0.45 & NS \\
\hline Quartile change & & $-1.15,0.45$ & $-1.6,0.05$ & & $-0.50,0.80$ & $-1.35,1.95$ & \\
\hline
\end{tabular}

FP400 = fluticasone propionate $400 \mu \mathrm{g} /$ day $(200 \mu \mathrm{g}$ bd); FP750 = fluticasone propionate $750 \mu \mathrm{g} /$ day $(375 \mu \mathrm{g}$ bd); BDP800 = beclomethasone dipropionate $800 \mu \mathrm{g} /$ day $(400 \mu \mathrm{g} \mathrm{bd}) ; \mathrm{BDP} 1500=$ beclomethasone dipropionate $1500 \mu \mathrm{g} /$ day $(750 \mu \mathrm{g} \mathrm{bd}) ; \mathrm{PICP}=$ procollagen type I carboxy terminal propeptide; ICTP = carboxy terminal cross linked telopeptide of type I collagen.

${ }^{\star} \mathrm{p}$ value $\mathrm{FP}-\mathrm{BDP}=$ significance derived from Wilcoxon rank sum test (difference between treatments in change from baseline). 
patients taking that dose of FP and none of the changes was greater than $-1 \%$. It is therefore likely that the result was of statistical but not clinical significance.

The results for the pQCT measurement at baseline and after 12 months of treatment are expressed as Z scores-that is, in terms of standard deviations above or below the predicted normal value for patients of the comparable age and sex (table 3). Mean baseline $\mathrm{Z}$ scores were at or near normal for all groups with little change after 12 months of treatment.

Statistical analyses of the changes from baseline in bone density of DXA lumbar vertebrae showed no differences between either of the two treatment comparisons at six months (table 4), nor was there any difference at 12 months between patients taking FP or BDP in the high dose comparison. In the low dose comparison, however, there was evidence of a statistically significant difference between treatments, patients taking BDP $800 \mu \mathrm{g} /$ day showing a negative change from baseline compared with those taking FP $400 \mu \mathrm{g} /$ day (table 4).

In addition, there was no significant difference in the median change from baseline in bone mineral content of the lumbar spine for either of the two treatment comparisons (low and high dose groups) at six and 12 months.

BONE MARKER DATA

The median baseline values in each treatment group and the normal range of the serum and urine markers of bone metabolism are shown in table 5. With the exception of the bone resorption marker urine phosphate, all median baseline values for all parameters were within the normal range in all treatment groups. Several patients had low baseline values for bone resorption markers (table 5), but these reductions were not correlated with any changes in bone formation markers nor were they clinically significant.

No consistent pattern emerged from the analysis of changes from baseline in markers of bone formation and resorption after six and 12 months of treatment. In the low dose comparison, a statistically significant difference in the change from baseline in osteocalcin at 12 months $(p=0.047)$ suggested lower bone formation activity in patients taking BDP $800 \mu \mathrm{g} /$ day than in those taking FP $400 \mu \mathrm{g} /$ day. Likewise, in the high dose comparison a statistically significant difference from baseline in the bone resorption marker ICTP at six months $(p=0.031)$ suggested greater bone

Table 6 Morning geometric mean serum cortisol concentrations (nmolll)

\begin{tabular}{clllll}
\hline & $\begin{array}{l}\text { Reference } \\
\text { range }\end{array}$ & FP400 & BDP800 & FP750 & BDP1500 \\
\hline Baseline & $138-635$ & 466 & 474 & 424 & 370 \\
n & & 22 & 21 & 13 & 13 \\
CV(\%) & & 29 & 35 & 59 & 54 \\
months & 517 & 440 & 499 & 397 \\
n & & 22 & 20 & 12 & 13 \\
CV(\%) & 47 & 31 & 33 & 34 \\
12 months & & 532 & 486 & 299 & 406 \\
n & 21 & 19 & 12 & 12 \\
CV(\%) & 41 & 50 & 122 & 41 \\
\hline
\end{tabular}

FP400 = fluticasone propionate $400 \mu \mathrm{g} /$ day $(200 \mu \mathrm{g}$ bd $) ; \mathrm{FP} 750=$ fluticasone propionate $750 \mu \mathrm{g} /$ day $(375 \mu \mathrm{g} \mathrm{bd}) ; \mathrm{BDP} 800=$ beclomethasone dipropionate $800 \mu \mathrm{g} /$ day $(400 \mu \mathrm{g} \mathrm{bd}) ; \mathrm{BDP} 1500=$ beclomethasone dipropionate $1500 \mu \mathrm{g} / \mathrm{day}(750 \mu \mathrm{g} \mathrm{bd}) ; \mathrm{CV}=$ coefficient of variation. resorption activity in patients taking $\mathrm{FP}$ $750 \mu \mathrm{g} /$ day than in those taking BDP $1500 \mu \mathrm{g} /$ day. Nevertheless, absolute and percentage changes from baseline for each bone marker in each patient offered no evidence of any clinically significant changes.

LUNG FUNCTION

No formal statistical analysis was performed on the lung function data. However, mean daily morning and evening PEF values taken for two weeks before each clinic visit and mean $\mathrm{FEV}_{1}$ values taken at bimonthly intervals throughout the 12 month study showed that the patients were well controlled on all treatments. Mean values either remained similar or tended to increase slightly above baseline values.

SAFETY

Adverse events were reported by a similar number of patients in both treatment groups. Overall, the adverse event profile was highly comparable between the two treatment groups and the events themselves were not unexpected in this group of patients. The most common events were infections of the upper respiratory tract and rhinitis. There were no reports of serious adverse events and the only withdrawal was due to pregnancy (one patient taking BDP $1500 \mu \mathrm{g} /$ day). The only predictable adverse event was hoarseness/dysphonia reported by three patients (one in each of the FP400, BDP800, and FP750 groups). There were no reports of allergic skin reactions, oral candidiasis, or rash/skin eruptions.

There were few reports of asthma exacerbations during the study. As expected, the incidence of exacerbations was slightly higher in the patients with more severe asthma in the high dose comparison. Nevertheless, the exacerbation rate was not statistically different between treatments. There was nothing to suggest that any of the study treatments had any effect on laboratory data or vital signs. Additionally, irrespective of treatment or dose, all geometric mean cortisol values remained within the normal range throughout the 12 month study period (table 6).

\section{Discussion}

The results of this study in a specific population of asthmatic patients provide reassuring prospective one year data indicating a lack of any effect of low and high doses of inhaled corticosteroids on bone mass and bone metabolism. Regular use of FP $(400 \mu \mathrm{g} /$ day and $750 \mu \mathrm{g} /$ day $)$ or BDP $(800 \mu \mathrm{g} /$ day and $1500 \mu \mathrm{g} /$ day) over a 12 month period in patients aged $20-55$ years with expected peak bone mass had no clinically significant effect on bone density or bone metabolism. The study also provided no evidence of any important differences between FP $400 \mu \mathrm{g}$ and BDP $800 \mu \mathrm{g}$ or between FP $750 \mu \mathrm{g}$ and BDP $1500 \mu \mathrm{g}$ daily. In addition, all treatments were well tolerated and mean morning cortisol concentrations remained within normal limits throughout the study in both the low and high dose groups. Lung function data showed that 
asthma was consistently well controlled in all patients, irrespective of treatment group, with a correspondingly low rate of asthma exacerbations.

A few isolated statistically significant differences were detected in the secondary safety parameters but these followed no consistent pattern and are unlikely to be of any clinical consequence. At 12 months bone density of the lumbar spine and osteocalcin were reduced in patients taking BDP $800 \mu \mathrm{g}$ and compact bone density of the proximal radius was reduced in patients taking FP $750 \mu \mathrm{g}$; ICTP absorption activity was greater at six months. However, these were isolated changes and should be interpreted with caution given the large number of statistical tests performed.

The findings of the present study largely concur with those of another recent longitudinal study involving 306 patients treated for six months with FP in doses of 500,750 , or $1000 \mu \mathrm{g} /$ day or BDP in doses of 1000,1500 , or $2000 \mu \mathrm{g} /$ day. ${ }^{18}$ The study recorded improved osteocalcin levels and, interestingly, even improved bone mineral densities in the spine, femoral neck and Ward's triangle, suggesting higher bone formation in the patients on FP than in those on BDP. Markers of bone resorption were similar with both treatments. These findings were in accordance with a second study involving 21 adults treated with BDP $1500 \mu \mathrm{g} /$ day or FP $750 \mu \mathrm{g} /$ day for six weeks. ${ }^{15}$ In comparison with these two studies we were unable to detect such a consistent pattern with regard to the effects of $\mathrm{FP}$ and $\mathrm{BDP}$ on bone markers and bone mineral density.

While there are considerable data on the effects of inhaled corticosteroids on bone density, ${ }^{31}$ the data tend to be inconsistent largely as a result of differences in the methodology of the studies involved. In some cases the primary assessment parameter has been biochemical markers of bone metabolism, in others it has been bone densitometry. The most useful biochemical markers, however, remain to be defined, ${ }^{15}{ }^{16}$ as does their relevance to changes in bone mass, particularly long term. ${ }^{817}$ Moreover, the results of many studies have been flawed through failure to control factors likely to affect bone density, principally past use of oral steroids, age, hormonal status, and diet.

The present study was designed to address some of the shortcomings of previous research. The study was conducted in patients of a specific age range with expected peak bone mass (men 20-55 years, women 20-45 years (all premenopausal)). Patients who had used more than three short courses of systemic oral corticosteroids in the last year or within the last eight weeks were excluded. Bone density was assessed by means of two complementary techniques, $\mathrm{pQCT}$ and DXA, selected because they measure different types of bone (trabecular, cortical and integral) at various anatomical sites. pQCT was particularly valuable since it enabled a separate measurement of trabecular bone which is the type of bone most affected by the osteopenic effects of corticosteroids. ${ }^{32}$
DXA is a widely used technique for measurement of the lumbar spine.

One limitation of the present study is that it did not control for the effects of physical activity on bone density in the different patient groups. While immobilisation was a specific reason for exclusion, it is likely that the degree and duration of the asthma suffered by most of the patients (longer than 12 years) may well have restricted their physical activity. The degree of restriction, however, was probably comparable across all treatment groups, given that exercise induced asthma was recorded in $68 \%$ of the participants and in a similar proportion in each treatment group.

A further limitation of the study is that, while it reached the desired statistical power by virtue of 23 patients randomised to each treatment regimen in the low dose comparison, insufficient patients were recruited into the high dose comparison. The analysis of the high dose group of patients was therefore underpowered so that no firm conclusions can be drawn from it. However, there were no obvious trends or consistent patterns to the data to indicate any cause for concern in the high dose group.

The determinants of corticosteroid induced osteoporosis are not completely understood. ${ }^{32}$ Individual or genetic susceptibility to bone loss and baseline bone density prior to treatment with oral or inhaled steroids may well influence any changes in bone density seen. ${ }^{31}$

However, this study provides reassuring prospective one year data showing that inhaled corticosteroids, in the range of doses used, had no adverse effects on bone mass and metabolism in this group of asthmatic patients.

The authors would like to thank the following investigators and their staff for their participation in this study: Dr H P Anderhub, their staff for their participation in this study: Dr H P Anderhub, Dr P Berg, Dr R Bezel, Dr M Ermanni, Dr M Heitz, Dr U
Honegger, Dr E Imhof, Dr U Lagler, Dr P Langloh, Dr A ZulHonegger, Dr E Imhof, Dr U Lagler, Dr P Langloh, Dr A Zul-
lig. Volumatic companies.

Funding: This study was sponsored by a grant from Glaxo Wellcome Research and Development, UK.

Conflict of interest: none.

1 Adinoff $\mathrm{AD}$, Hollister JR. Steroid induced fractures and bone loss in patients with asthma. $N$ Engl $\mathcal{f}$ Med 1983;309:265-8

2 Geddes DM. Inhaled corticosteroids: benefits and risks. Thorax 1992;47:404-7.

3 Packe GE, Douglas JG, McDonald AP, et al. Bone density in asthmatics taking high dose inhaled beclomethasone dipropionate. Thorax 1992;47:415-8.

4 Hanania NA, Chapman KR, Sturtridge WC, et al. Dose-related decrease in bone density among asthmatic patients treated with inhaled corticosteroids. $\mathcal{f}$ Allergy Clin Immunol 1995;96:571-9.

5 Ip M Lam K, Yam L, Kung A, et al. Decreased bone mineral density in premenopausal asthma patients receiving lonsity in premenopausal asthma patients receiv

6 Toogood JH, Baskerville JC, Markov AE, et al. Bone mineral Toogood JH, Baskerville JC, Markov AE, et al. Bone mineral
density and the risk of fracture in patients receiving long-term inhaled steroid therapy for asthma. F Allergy Clin Immunol 1995;96:157-66.

7 Packe GE, Robb O, Robins SP, et al. Bone density in asthmatic patients taking inhaled corticosteroids: comparison of budesonide and beclomethasone dipropionate. $\mathcal{F} R$ Coll Physicians (London) 1996;30:128-32.

8 Boulet LP, Giguére MC, Milot J, et al. Effects of long-term use of high-dose inhaled steroids on bone density and calcium metabolism. F Allergy Clin Immunol 1994;94:796803.

9 Wolff A, Adelsberg B, Aloja J. Effect of inhaled corticosteroid on bone density in asthmatic patients: a pilot study. Ann Allergy 1991;67:117-21.

10 Herrala, Puolijoki H, Impivaara O, et al. Bone mineral density in asthmatic women on high-dose inhaled beclometha-

11 Teelucksingh S, Padfield PL, Tibi L, et al. Inhaled corticosteroids, bone formation and osteocalcin. Lancet 1991;338:60-1. 
12 Pouw EP, Prummel MF, Ossting $\mathrm{H}$, et al. Beclomethasone inhalation decreas serum osteocalcin concentrations. inhalation decreases

13 Ali NJ, Capewell S, Ward MJ. Bone turnover during high dose inhaled corticosteroid therapy. Thorax 1991;46:1604.

14 Hodsman AB, Toogood JH, Jennings SB, et al. Differential effects of inhaled budesonide and oral prednisolone on serum osteocalcin. f Clin Endocrinol Metab 1991;72:53040.

15 Bootsma GP, Dekhuijzen PNR, Festen J, et al. Fluticasone propionate does not influence bone metabolism in contrast 1996;153:924-30.

16 Birkebaek N H, Esberg G, Andersen K, et al. Bone and collagen turnover during treatment with inhaled dry powder lagen tunide and beclomethasone dipropionate. Arch Dis Child 1995;73:524-7.

17 Kerstjens HAM, Postma DS, van Doormaal JJ, et al. Effects of short term treatment with inhaled corticosteroids on bone term treatment with inhaled corticosteroids on rax 1994;49:652-6.

18 Pauwels RA, Yernault JC, Demedts MS, et al on behalf of the Belgian Multicentre Study Group. Safety and efficacy of fluticasone and beclomethasone in moderate to severe asthma. Am F Respir Crit Care Med 1998;157:827-32.

19 Holliday SM, Faulds D, Sorkin M. Focus on inhaled fluticasone proprionate. Drugs 1994;47:318-31.

20 Harding SM. The human pharmacology of fluticasone propionate. Respir Med 1990;84(Suppl A):25-9.

21 Barnes N, Harris TAJ, Hallet C. Clinical experience with fluticasone propionate in asthma: a meta-analysis of efficacy and systemic activity compared with budesonide and beclomethasone dipropionate at half the microgram dose or less. Respir Med 1998;92:95-104.

22 Fabbri L, Burge PS, Croonenborgh L, et al. Comparison of fluticasone propionate with beclomethasone dipropionate in moderate to severe asthma treated for one year. Thorax in moderate to seve.
23 Hoekx JCM, Hedline G, Pedersen W, et al. Fluticasone propionate compared with budesonide: a double-blind trial in asthmatic children using powder devices at a dosage of 400 asthmatic children using powder devices

24 Lundback B, Alexander M, Day J, et al. Evaluation of fluticasone propionate $(500 \mathrm{mcg} / \mathrm{day})$ administered either as dry powder via a Diskhaler inhaler or pressurised inhaler and compared with beclomethasone dipropionate $(1000 \mathrm{mcg} / \mathrm{day})$ administered by pressurised inhaler. Respir Med 1993;87:609-20.

25 Barnes NC, Marone G, Di Maria GU, et al. A comparison of fluticasone propionate $1 \mathrm{mg}$ daily with beclomethasone dipropionate $2 \mathrm{mg}$ daily in the treatment of severe asthma. Eur Respir f 1993;6:877-84.

26 Langdon CG, Thompson J. A multicentre study to compare the efficacy and safety of inhaled fluticasone propionate and budesonide via metered dose inhalers with mild-tomoderate asthma. Br f Clin Res 1994;5:73-84.

27 Connolly A. A comparison of fluticasone propionate $100 \mu \mathrm{g}$ twice daily with budesonide $200 \mu \mathrm{g}$ twice daily via their respective powder inhaler devices in the treatment of mild asthma. Eur 7 Clin Res 1995; 7:15-29.

28 Rüegsegger P, Kalender WA. A phantom for standardization and quality control in peripheral bone measurements by pOCT and DXA. Phys Med Biol 1993;38:1963-70.

29 Kalender WA. A phantom for standardization and quality control in spinal bone mineral measurements by QCT and DXA: design consideration and specifications. Med Phys DXA: design cons

30 Rüegsegger P, Medici TC, Anliker M. Corticosteroid induced bone loss. A longitudinal study of alternate day therapy in patients with bronchial asthma using quantitative computed tomography. Eur f Clin Pharmacol 1983;25: $615-20$

31 Efthimiou J, Barnes PJ. Effect of inhaled corticosteroids on bones and growth. Eur Respir f 1998;11:1167-77.

32 Baylink DJ. Glucorticoid-induced osteoporosis. N Engl f Med 1983:309:306-8. 